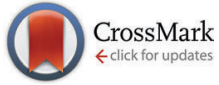

Cite this: New J. Chem., 2014, 38, 4922

Received (in Montpellier, France) 3rd June 2014,

Accepted 22nd July 2014

DOI: $10.1039 / c 4 n j 00907 j$

www.rsc.org/njc

\section{Stable graphite exfoliation by fullerenol intercalation via aqueous route}

\author{
Rachana Kumar, ${ }^{* a}$ Pramod Kumar, ${ }^{* b}$ Samya Naqvi, ${ }^{a}$ Neha Gupta, ${ }^{a}$ Niharika Saxena, ${ }^{a}$ \\ Jitendra Gaur, ${ }^{a}$ Jitendra K. Maurya ${ }^{C}$ and Suresh Chand ${ }^{a}$
}

\begin{abstract}
Graphene is a wonder material possessing unique properties; however, graphene prepared by exfoliation of graphite has property to restack because of van der Waals interactions to form graphite. This restacking can be prevented by insertion of large molecules like fullerene, which not only exfoliates graphite layer but also prevents restacking of prepared graphene sheets. The present article also describes a mild method of graphite oxide synthesis (GO) for lower degree of oxidation resulting in less defected (ruptured carbon framework) graphene sheets. Exfoliation is performed by intercalation of large fullerene molecules by aqueous reaction of fullerene hydroxide (fullerenol) with the epoxy functionalities on graphite oxide to prepare fullerene intercalated graphite (G-Fol). Fullerene functionalization of GO to form G-Fol has been established by FTIR spectroscopy, UV-Vis spectroscopy, TGA and number of layers has been ascertained by Raman spectroscopy, XRD and HRTEM. Stable exfoliation of G-Fol has been confirmed by change in absorbance with time. Photoluminescence property of the material is also evaluated by fluorescence emission and excitation measurement at different excitation and emission wavelengths, respectively. The present article explains a new method of exfoliation of graphite to form stable functionalized graphene layers with fewer defects for future applications as buffer layer in electronic devices.
\end{abstract}

\section{Introduction}

Graphene is single layer graphite, composed of a honeycomblike arrangement of carbon atoms, and it has applications in several areas. $^{1-12}$ Many efforts have been made to take advantage of these exceptional properties for the electronic and energy applications. ${ }^{13-17}$ Graphene is prepared from graphite by several methods, ${ }^{18-20}$ among which, single layer graphene prepared by 'scotch-tape' method has shown promising electrical properties that could be useful for developing novel electronic devices. ${ }^{21-24}$ To prepare graphene materials of different sheet size, functionalities and structures, chemical route through oxidation of graphite is being widely used. ${ }^{25-28}$ The rationale is the easy scalability of the process and the cost involved. Nowadays, the most widely used method for preparation of graphite oxide and or graphene oxide is either Brodie's ${ }^{25}$ or the Hummers ${ }^{26}$ method. These methods, involve reaction of graphite with strong oxidizing agents, like sulphuric acid, nitric acid in presence of salts, like potassium chlorate or potassium

\footnotetext{
${ }^{a}$ Physics of Energy Harvesting Division, National Physical Laboratory, Dr K. S. Krishnan Marg, New Delhi, 110012, India.

E-mail: rachanak@nplindia.org; Fax: +91 114560 9310; Tel: +91 1145609353

${ }^{b}$ Department of Physics, Indian Institute of Information Technology Allahabad,

Uttar Pradesh, 211012, India. E-mail: pkumar@iiita.ac.in; Tel: +91 5322922202

${ }^{c}$ Department of Chemistry, Delhi University, New Delhi, 110007, India
}

permanganate, and lot of precautions are required during the addition of salts. Introduction of oxygen containing functional groups help in increasing the $d$-spacing of GO as well as change the hybridization from $\mathrm{sp}^{2}$ to $\mathrm{sp}^{3}$. Furthermore, ultrasonication followed by ultra-centrifugation helps in exfoliation of graphite oxide and formation of graphene layers. It is seen that the resultant graphene sheets have permanent defects, such as the partial cleavage of the hexagonal framework of planar carbon atoms, which cannot be healed and tend to reattach to form graphite through van der Waals interactions. ${ }^{28,29}$

Recently, a wet chemical approach has been introduced to prepare graphene from GO with the carbon skeleton preserved in the order of tens of nanometers. ${ }^{29}$ There are also several other routes for graphite exfoliation by insertion of organic molecules to avoid such agglomeration and also to prepare less defected graphene sheets. Graphene has been functionalized with several types of molecules, like polymers, small molecules, porphyrin, diazonium salts, and alkyl isocyanates. ${ }^{30-37}$ Some research has also been carried out to attach bulky fullerene molecules in between the graphite layers to exfoliate them, like pyrrolidine fullerene ${ }^{38}$ or methano fullerene. ${ }^{39-44}$ In some of the above cases, fullerene molecules were only attached on the edges. Zhang et al., reported the exfoliation of graphite to graphene monolayers by inserting fullerene molecules in between graphene layers of graphite and grafted on both sides of graphene. ${ }^{40}$ The grafted fullerene exfoliate graphene sheets 
to higher extent and avoid the restacking and also help in maintaining the large surface area.

In the present article, we have further simplified the insertion of fullerene molecules between graphene layers by very simple aqueous route where graphite oxide (GO) is prepared by oxidation with conc. nitric acid. ${ }^{45}$ Synthesis of graphite oxide without use of strong salts avoids the impurities of salt byproducts. ${ }^{46}$ On the other hand, water soluble polyhydroxyl fullerene (fullerenol) was prepared by the modified base method. ${ }^{47-50}$ Under basic condition, the strong electrophilic character of fullerene cage easily releases protons to form fulleroxide anions, (Fol-O) ${ }^{-n}$, which is capable of undergoing several types of reactions. Fullerenol undergoes nucleophilic addition reactions on epoxy groups to form star-like macromolecules under mild basic condition. ${ }^{50}$ Here also, we have exploited the strong nucleophilic character of the sodium salt of fullerenol to undergo nucleophilic addition reaction with epoxy groups on the surface of GO, resulting in chemically bonded fullerene functionalized graphene sheets (G-Fol).

The resultant G-Fol was characterized for its optical properties and thermal stability, and also the chemical attachment was established by FTIR spectroscopy. In contrast to the previous reports for edge functionalization of graphite oxide on reaction with fullerenol molecules, in the present case, we have obtained stable exfoliation with formation of few layered (2-4) graphene sheets. ${ }^{43}$ The quality, exfoliation and number of layers of thus prepared graphene have been confirmed by powder X-ray diffraction (XRD) and Raman spectroscopy measurements. To confirm the stability of dispersion of the exfoliated graphite, optical studies have been performed with the stable supernatant of the dispersions in different solvents. Graphite exfoliation was further confirmed by high-resolution transmission electron microscopy.

\section{Experimental section}

Graphite oxide (GO) was prepared by refluxing micrometer sized-graphite in conc. nitric acid $\left(1 \mathrm{mg} \mathrm{mL}{ }^{-1}\right)$ for 24 hours (Scheme 1). The resultant material was filtered and washed several times with deionized water to remove all acid impurities and dried in oven at $80{ }^{\circ} \mathrm{C}$. Fullerenol (Fol) was prepared by the reaction of aqueous solution of sodium hydroxide $(3 \mathrm{~g})$ with toluene solution of fullerene $(50 \mathrm{mg})$ in presence of phase transfer catalyst (tetra butyl ammonium bromide). The clear toluene layer was separated and brown aqueous layer was precipitated with the addition of excess methanol. The brown product was collected by centrifugation and washed several times with methanol/water mixture $(80: 20)$ to remove the traces of sodium hydroxide. Thus, the prepared fullerenol was further reacted with GO by a simple aqueous route. Sodium salt solution of fullerenol ( $45 \mathrm{mg}$ ) was prepared in water, added to aqueous suspension of GO and first stirred at room temperature for 24 hours followed by heating at $50{ }^{\circ} \mathrm{C}$ for 12 hours. The resultant suspension was washed with cold water to remove unreacted fullerenol and the resultant material (G-Fol) was dried under vacuum at $60{ }^{\circ} \mathrm{C}$ for 12 hours.

Thus, synthesized G-Fol was characterized by Fourier transform infrared spectroscopy (FTIR) using $\mathrm{KBr}$ pellets on Perkin Elmer FTIR Spectrum 2. FTIR spectra were collected over a range from 3500 to $500 \mathrm{~cm}^{-1}$. A background spectrum in air was collected before scanning the samples. UV-vis spectroscopy measurement was performed on a Shimadzu UV-vis spectrophotometer in solution. Emission and excitation fluorescence measurements were performed on Varian (CARY Eclipse) fluorescence spectrophotometer in THF suspension. Horiba Jobin Yvon (FluoroHub) was used to record time resolved fluorescence. Thermal gravimetric analysis (TGA) was run under nitrogen flow of $20 \mathrm{~mL} \mathrm{m^{-1 }}$ using Perkin Elmer (Pyris 1) TGA instrument and mass loss was recorded as a function of temperature. The samples were heated from room temperature to $950{ }^{\circ} \mathrm{C}$ at a ramp rate of $10{ }^{\circ} \mathrm{C} \mathrm{min}^{-1}$. Raman spectroscopy was performed on a Renishaw Raman Microscope in powder samples. GO and G-Fol were also characterized by XRD on Rigaku diffractometer with $\mathrm{Cu}-\mathrm{K} \alpha$ radiation $(\lambda=1.54056 \AA)$ to estimate the interlayer distances. Cyclic voltammetry measurements were performed using a three electrode standard configuration with a platinum wire as counter electrode and $\mathrm{Ag} / \mathrm{AgCl}$ sat. as reference electrode in a $0.05 \mathrm{M}$ TBAPF6 (tetra- $n$ butylammonium hexafluorophosphate) and acetonitrile solution as electrolyte. A film of GO/G-Fol was prepared on working electrode (Pt disc) by drop casting. The solution was purged with $\mathrm{N}_{2}$ for at least 30 min before measurements. Current versus voltage was measured on an Autolab potentiostat. G-Fol was also characterized by HRTEM (Technai $\mathrm{G}^{2} \mathrm{~F} 30, \mathrm{HV}=300.0 \mathrm{kV}$ ) to observe the exfoliation and formation of graphene sheets.

\section{Results and discussions}

In the present work, graphite oxide (GO) is prepared by a milder method and exfoliation has been performed by fullerene functionalization following a very simple chemical route by nucleophilic addition of fulleroxide anion on the epoxy groups of GO. This is a facile and friendly route for graphite exfoliation by

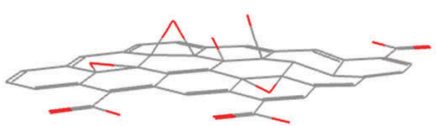

GO

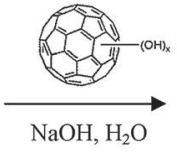

$\mathrm{NaOH}, \mathrm{H}_{2} \mathrm{O}$

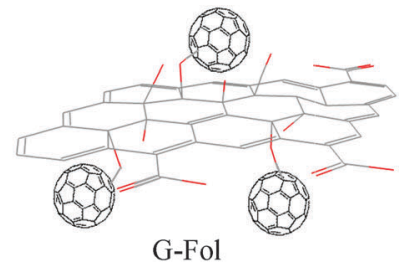

Scheme 1 Synthesis of fullerenol intercalated graphene by the reaction of $\mathrm{GO}$ with $\mathrm{NaOH}$ solution of fullerenol. 


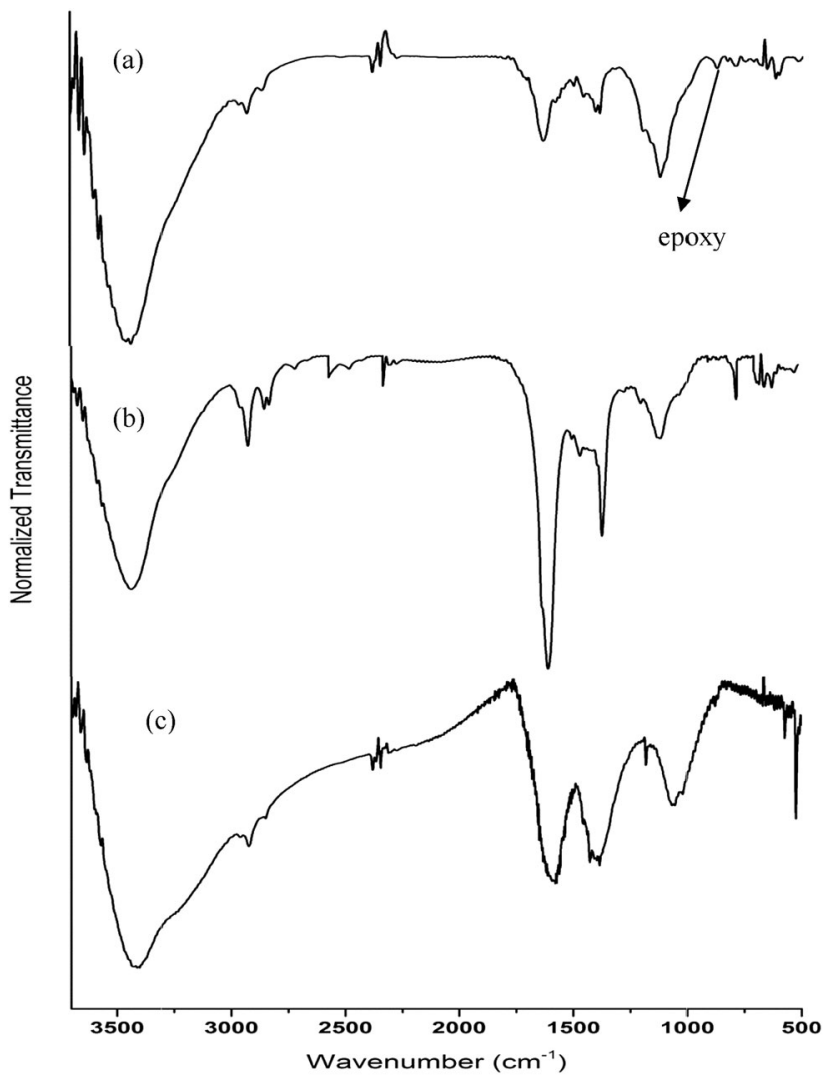

Fig. 1 FTIR spectra of (a) GO, (b) G-Fol and (c) fullerenol.

inserting fullerenol molecules to weaken the van der Waals forces between the neighbouring graphene sheets. The detailed reaction steps are shown in Scheme 1. The resultant samples (Fol, GO and G-Fol) were characterized by FTIR for the chemical functionalities present in the molecules (Fig. 1). FTIR spectra of graphite oxide clearly showed the appearance of peaks at $878 \mathrm{~cm}^{-1}$ (epoxy group), $1399 \mathrm{~cm}^{-1}$ (C-OH), and small peak at $1723 \mathrm{~cm}^{-1}$ for $\mathrm{C}=\mathrm{O}$ of carboxylic acid group (Fig. 1a). Peak at $1630 \mathrm{~cm}^{-1}$ can be assigned to adsorbed water molecules. On chemical attachment of fullerenol molecules, typical epoxy peak disappeared with appearance of broad $\mathrm{O}-\mathrm{H}$ stretching peak at $3439 \mathrm{~cm}^{-1}, \mathrm{C}=\mathrm{C}$ stretching peak at $1611 \mathrm{~cm}^{-1}$ (shifted from $1590 \mathrm{~cm}^{-1}$ in fullerenol, Fig. 1c), $1120 \mathrm{~cm}^{-1}$ for $\mathrm{C}-\mathrm{O}$ stretching and distinct peak of $\mathrm{C}=\mathrm{O}$ stretching at $1743 \mathrm{~cm}^{-1}$ in G-Fol samples (Fig. 1b). Thus, the FTIR results clearly indicate the successful chemical attachment of fullerenol molecules on GO.

A comparative UV-vis analysis was performed for the suspended samples of GO and G-Fol in THF (Fig. 2). Both the samples were sonicated for an hour before recording the data. GO showed the characteristic absorption bands at 285, 300, 515 and $560 \mathrm{~nm}$, while in G-Fol an additional absorption band was observed at $336 \mathrm{~nm}$, which is characteristic of fullerene derivatives. ${ }^{51}$

TGA has been exploited as a tool to calculate the number of hydroxyl groups or addends (bulky substituents) attached exohedrally on fullerene ball. ${ }^{52,53}$ In the present work, the number of hydroxyl groups per fullerene has been calculated to be $\sim 16$.

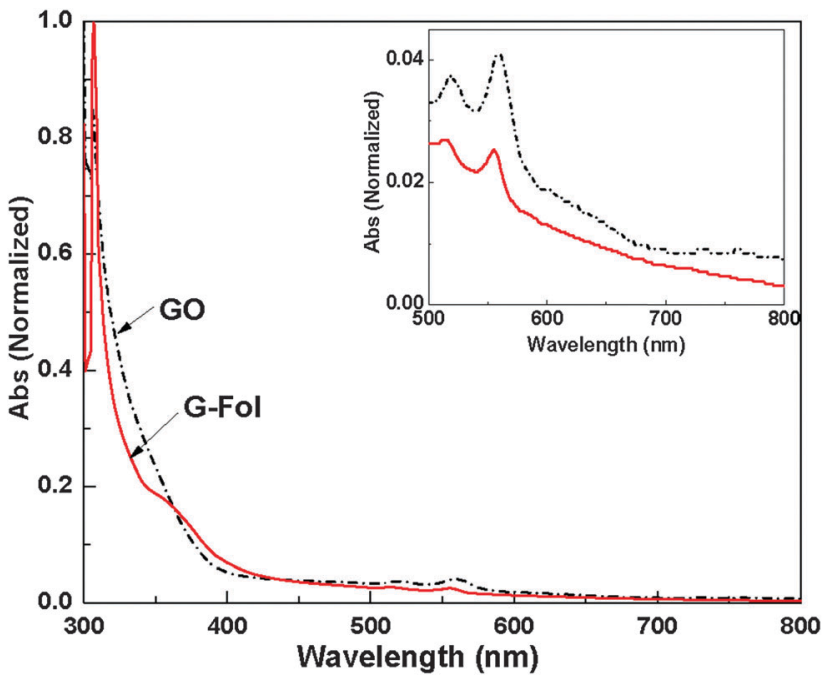

Fig. 2 UV-vis absorption spectra of GO and G-Fol samples in THF. Inset shows the $10 \times$ view of the $500-800 \mathrm{~nm}$ region.

TGA thermogram of G-Fol and its comparison with unreacted fullerenol and GO reveals several interesting observations. The analysis ascertains the chemical attachment of fullerenol molecules onto graphene sheets and also predicts its thermochemical behaviour. The typical thermogram of GO, fullerenol, and G-Fol are depicted in Fig. 3. No major weight loss was observed for GO $(\sim 4.2 \%)$ samples up to $950{ }^{\circ} \mathrm{C}$, and it only showed a small weight loss at $\sim 326{ }^{\circ} \mathrm{C}$ for the decomposition of weak bonds like epoxide (Fig. 3a). Compared to $\sim 50 \%$ weight loss for graphite oxide prepared by Hummers process, only $13 \%$ weight loss up to $950{ }^{\circ} \mathrm{C}$ attributes to the lower degree of oxidation of graphite sheets in the present case. ${ }^{54}$ On the other hand, fullerene intercalated graphite sample (G-Fol) showed continuous weight loss up to $950{ }^{\circ} \mathrm{C}$ (Fig. 3b) as in the case of fullerenol. The dehydroxylation of fullerenol starts at $\sim 150-160{ }^{\circ} \mathrm{C}$ and completes between the temperature range of $150-570{ }^{\circ} \mathrm{C}$ before the structural degradation of fullerene core starts (Fig. 3c). Similarly, for G-Fol, the first weight loss occurs between 163 and $550{ }^{\circ} \mathrm{C}\left(352{ }^{\circ} \mathrm{C}\right.$ is the crest temperature), which is for the loss of free hydroxyl groups of fullerenol. Another major loss starts from $680^{\circ} \mathrm{C}$ and only $66.8 \%$ residue is left after $950{ }^{\circ} \mathrm{C}$ compared to $88.3 \%$ for GO. The second weight loss is due to the breakage of graphite-O-fullerene bonds of G-Fol. Because of high functionality of graphite on chemical attachment of fullerenol molecules, a continuous decomposition occurs in TGA of G-Fol; however, this material is also showing better thermal stability compared to previously reported data for fullerene modified graphite. ${ }^{55}$ Thus, TGA results further confirm the successful functionalization of graphite with fullerenol molecules.

Graphite oxides prepared by other methods are highly oxidized material with high hydrophilic nature and readily exfoliate in water to yield stable dispersions, consisting mostly of single layered graphene oxide sheets. Paredes et al. ${ }^{54}$ have reported the dispersion behaviour of graphite oxide in different solvents and identified that graphite oxide is exfoliated into individual graphene oxide sheets or few layers sheets with long 

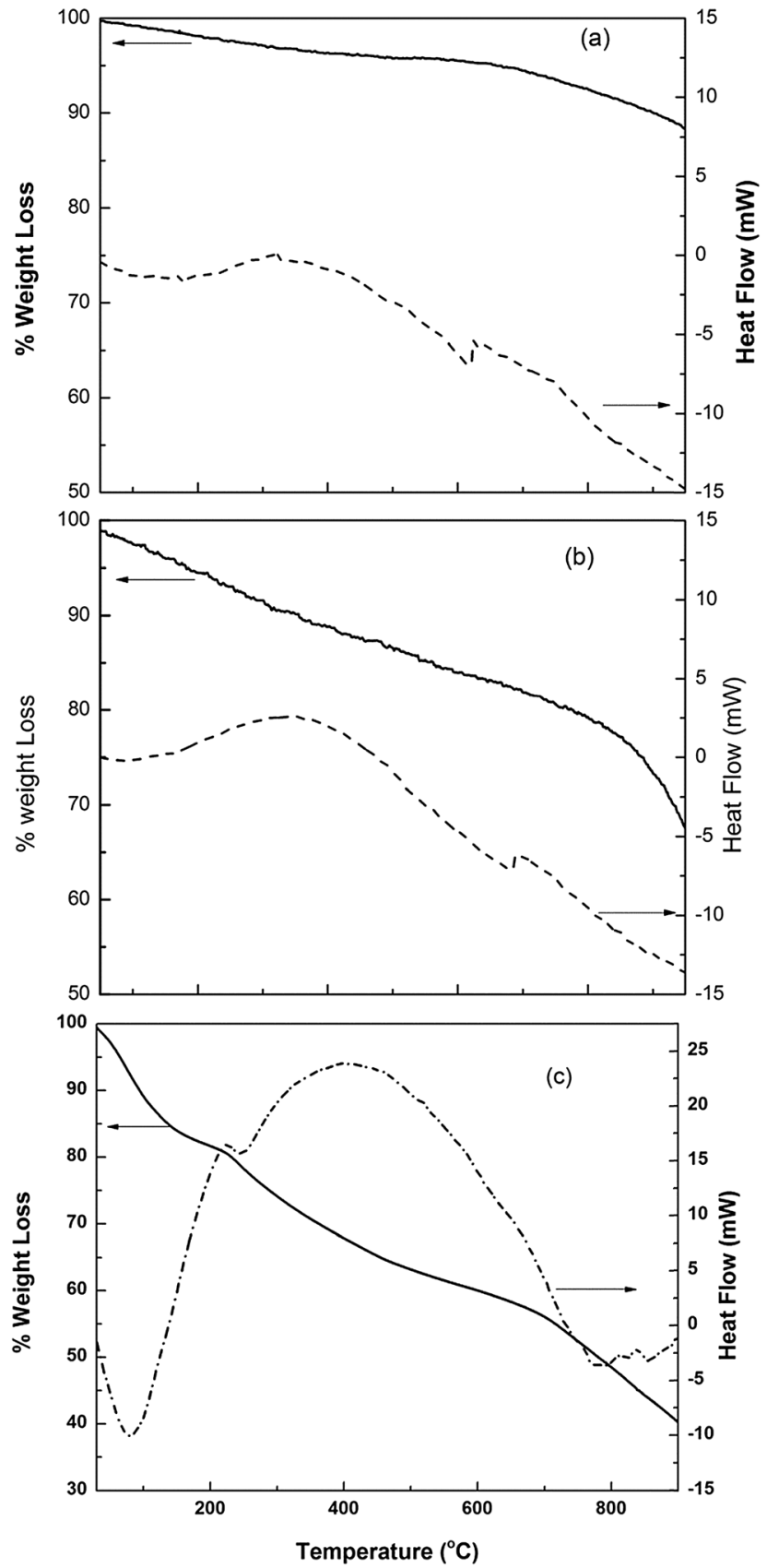

Fig. 3 TGA graph of (a) GO, and (b) G-fol and (c) fullerenol under $\mathrm{N}_{2}$.

term stability in several solvents. The stability of dispersion of GO and G-Fol samples in different polarity solvents was studied to determine the significance of such chemical modification of GO in exfoliation and dispersion stability in different solvents. GO was dispersed in five solvents $\left(0.5 \mathrm{mg} \mathrm{mL}^{-1}\right.$ in water, DMF, THF, ethanol, ethylene glycol) by ultrasonication. Fig. 4 shows the dispersed GO sample in different solvents. The supernatants after two weeks of dispersion were used to record the UV-vis spectra to compare the dispersion stability. Absorption spectra of the supernatant of GO and G-Fol in water are shown in Fig. 5a, where GO shows the onset at $\sim 425 \mathrm{~nm}$; however, G-Fol had extended absorption in the visible region, which is

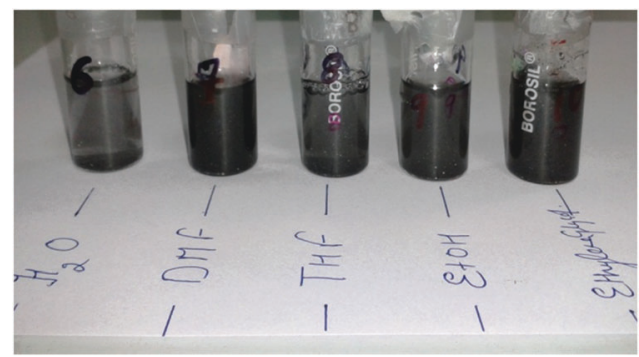

Fig. 4 Photograph of suspension in different solvents for GO.

the characteristic of the fullerene containing materials. ${ }^{51} \mathrm{G}-\mathrm{Fol}$ in water also showed the structured absorption bands at $273 \mathrm{~nm}$ for graphene and $356 \mathrm{~nm}$ for fullerene moieties, respectively. G-Fol showed higher absorption intensity in water showing better and stable dispersion compared to GO on attachment of fullerenol molecules with free hydroxyl groups helping in better dispersion. Similarly, G-Fol also showed better dispersion stability in THF than GO. On comparing the dispersion stability among the five solvents, DMF, ethanol and THF showed the highest and comparable stability for GO (Fig. 5c). Thus, GO and G-Fol samples dispersion with long term stability can be prepared in THF and water with best exfoliation. To further analyze the long term stability, the absorbance of supernatant of G-Fol in water was measured at different intervals, i.e., after three weeks, one month and two months of dispersion respectively without any further sonication (Fig. 5d). Only 25\% absorbance loss was seen even after two months, showing stability of the suspended graphene sheets avoiding the restacking.

To further confirm the graphite layer exfoliation by insertion of fullerenol molecules, Raman analysis was carried out (Fig. 6). Graphite oxide showed the characteristic D-band at $1360 \mathrm{~cm}^{-1}$, G-band at $1580 \mathrm{~cm}^{-1}$ and $2 D$-band at $2729 \mathrm{~cm}^{-1}$. On incorporation of fullerenol molecules by chemical bonding the overall $\mathrm{sp}^{3}$ carbon content has been increased (on the site of $-\mathrm{OH}$ groups in hydroxyl fullerene) in the material, resulting in more intense D-band (at $1355 \mathrm{~cm}^{-1}$ ) with appearance of additional peaks of fullerenol at $1261 \mathrm{~cm}^{-1}$ and $1399 \mathrm{~cm}^{-1}$. For proper comparison, Raman spectra of fullerenol was also recorded, showing three characteristic bands at $1387 \mathrm{~cm}^{-1}\left(\mathrm{sp}^{3}\right.$ hybridization), $1456 \mathrm{~cm}^{-1}$, and $1574 \mathrm{~cm}^{-1}$ ( $\mathrm{sp}^{2}$ hybridization). In G-Fol, the $1456 \mathrm{~cm}^{-1}$ band of fullerenol is shifted to lower energy and appears at $1399 \mathrm{~cm}^{-1}$, which is caused by the plausible charge transfer from fullerenol moiety to graphite sheets as fullereneoxide is strongly nucleophilic. Nevertheless, the $I_{\mathrm{D}} / I_{\mathrm{G}}$ ratio is very small, providing evidence of low degree of functionalization of graphene sheets conserving its defect free nature. On analyzing the 2D peak, the relative intensity of G/2D peak is found to be $\sim 0.7$ in G-Fol, indicating the formation of few layer graphene (2-4 layers). ${ }^{56}$ Moreover, the width of 2D peak is split into four sub-peaks on fitting, as shown in Fig. 6 (inset), which directly reflects the doublelayered graphene formation..$^{56,57}$

The lattice expansion of graphite on oxidation is one of the indications for the degree of oxidation. An XRD analysis has been performed on GO and G-Fol samples at ambient conditions 

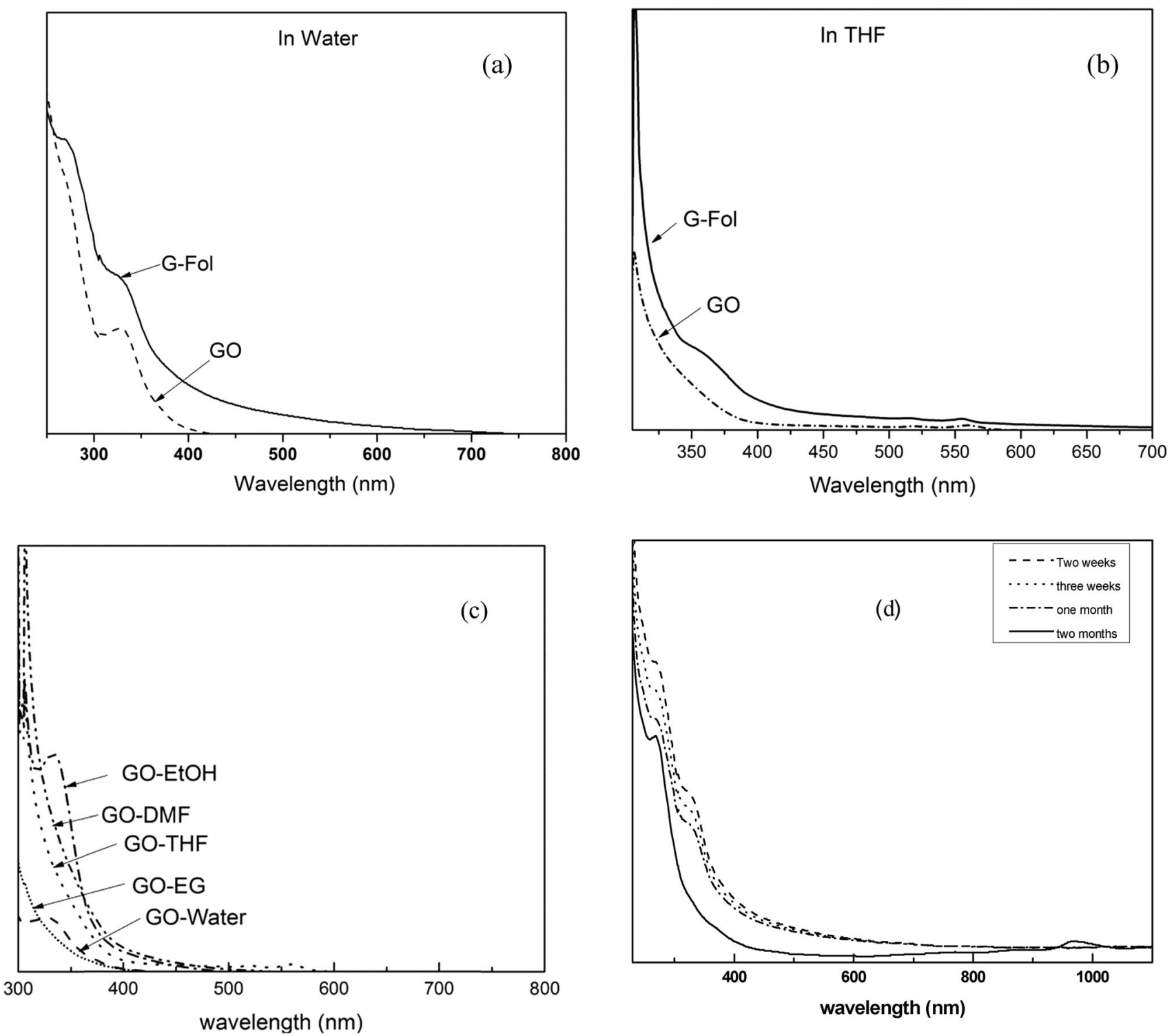

Fig. 5 Absorption spectra of stable suspension (a) of GO and G-Fol in water and (b) of GO and G-Fol in THF, and (c) GO in different solvents and (d) absorbance versus time curve for G-Fol in water.

to confirm the functionalization of graphene sheets and also the degree of functionalization. Fig. 7 shows the XRD pattern of powder sample of GO and G-Fol. As expected, the characteristic [001] diffraction peak of $\mathrm{GO}$ appears at $12.88^{\circ}(2 \theta)$, corresponding to an inter-layer distance of $6.88 \AA$, which is comparatively lower than the highly oxidized graphite oxide $(\sim 8 \AA)^{58}$ because of the lower degree of oxidation, corroborating with the Raman results. On functionalization with fullerenol molecules, the interlayer distance is further increased to $8.1 \AA$, indicating the exfoliation to few layers (2-4) graphene. The larger interlayer distance results in less ordered packing of sheets, which is evidenced by the significantly broader [001] peak in G-Fol. No peak appeared at $\sim 25^{\circ}(2 \theta)$, which also excludes the formation of any chemically converted graphite in unfunctionalized areas on the surface of graphene sheets.

Fluorescence spectra of GO and G-Fol were recorded in THF with the same sample prepared in absorption measurement experiment and are shown in Fig. 8. On excitation with $350 \mathrm{~nm}$ wavelength, GO shows three emission bands at 406, 572 and $618 \mathrm{~nm}$ for the three corresponding absorption bands at 308, 515 and $560 \mathrm{~nm}$. On the other hand, G-Fol sample shows four emission bands at 405 and $415 \mathrm{~nm}$ and highly blue-shifted bands at 560 and $605 \mathrm{~nm}$ compared to GO samples $(\sim 12 \mathrm{~nm}$ blue shift). Emission peak at $415 \mathrm{~nm}$ corresponds to absorption peak at $360 \mathrm{~nm}$ for the fullerene moiety in G-Fol sample. Because of the chemical modification of graphite oxide with fullerene, the intensity of graphite emission peaks at 560 and $605 \mathrm{~nm}$ are highly quenched and shifted to higher energy due to effective charge transfer in G-Fol.

Emission spectra of G-Fol was also recorded at different excitation wavelength in THF to study the effect of excitation energy on intensity of emission bands corresponding to fullerene and graphite moieties in $400 \mathrm{~nm}$ and $500-600 \mathrm{~nm}$ range. 


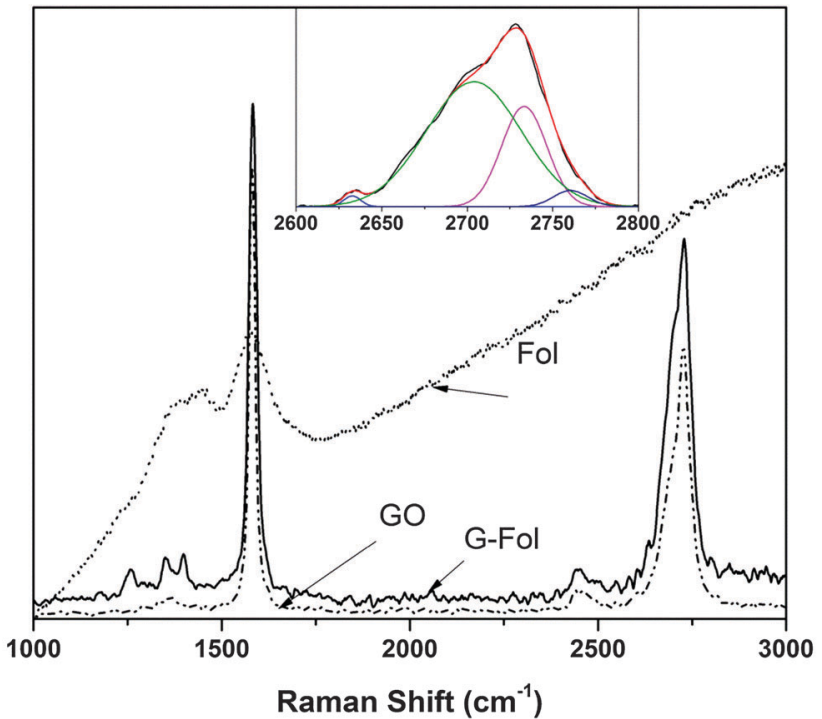

Fig. 6 Raman spectra of GO, fullerenol and G-Fol in solid state. The inset shows the Lorentzian fit for 2D peak of G-Fol in to four sub peaks.

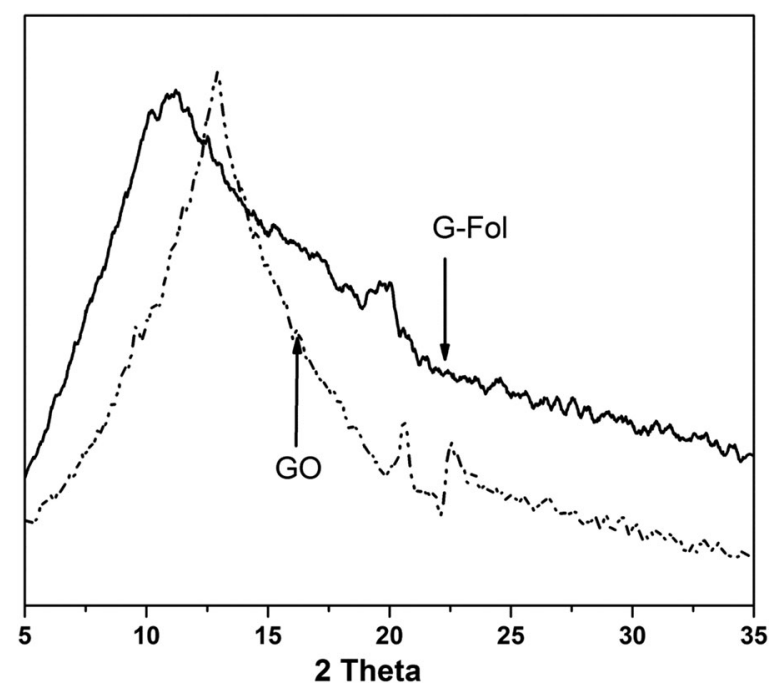

Fig. 7 Powder XRD patterns of GO and G-Fol.

As shown in Fig. 9, on excitation with energy lower than $3.5 \mathrm{eV}$ $(350 \mathrm{~nm})$, very weak emission was observed in both the regions. Emission peak intensity at $415 \mathrm{~nm}$ was highest with $350 \mathrm{~nm}$ excitation due to the highest absorption by fullerene in this range $\left(\lambda_{\max }=336 \mathrm{~nm}\right)$. On further lowering the excitation energy, the intensity of emission bands at 560 and $605 \mathrm{~nm}$ increases with the quenching of the $415 \mathrm{~nm}$ peak intensity, which also shows a red shift. Excitation with $2.47 \mathrm{eV}(500 \mathrm{~nm})$ shows the highest emission intensity for 560 and $605 \mathrm{~nm}$ bands corresponding to graphite sheet absorption in these regions. Thus, the emission fluorescence study of G-Fol sample gives good understanding of electron transfer processes on excitation with different energies and at the same time emission colour tuning by controlling the excitation energy.

To further analyze these data, excitation at different emission wavelengths were recorded (Fig. 10). For 350 and $400 \mathrm{~nm}$

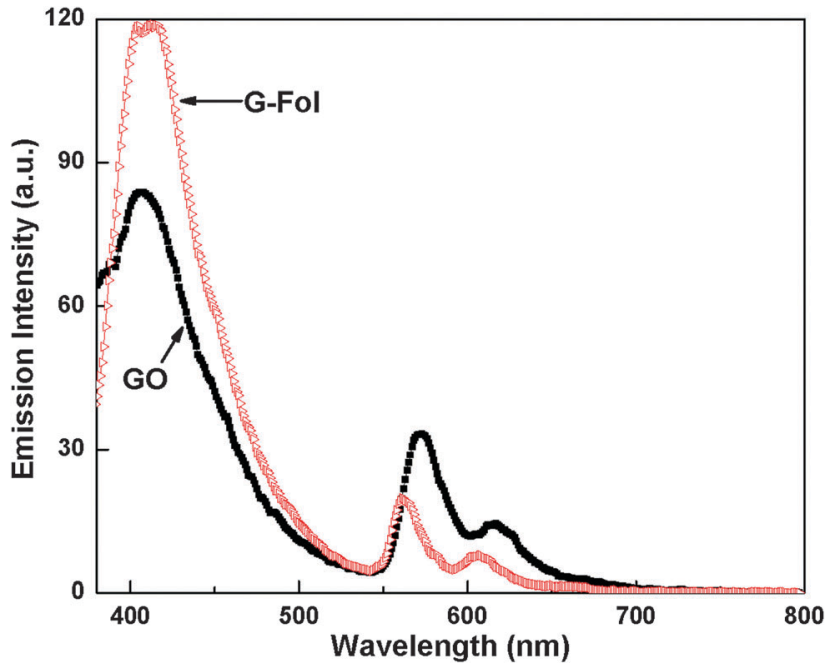

Fig. 8 Emission fluorescence spectra of GO, and G-Fol in THF with $350 \mathrm{~nm}$ excitation.

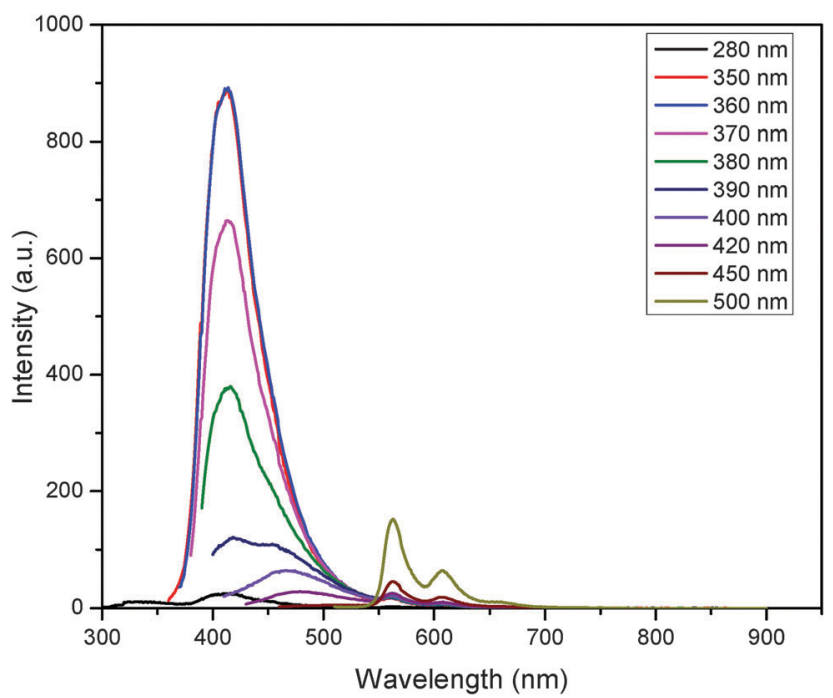

Fig. 9 Emission fluorescence spectra of G-Fol in THF at different excitation wavelengths.

emission wavelength, the highest excitation intensity was observed in the $300-400 \mathrm{~nm}$ range (corresponding to fullerene moiety). On further lowering the emission energy, excitation intensity in 500-600 $\mathrm{nm}$ goes up, which is very well understood as graphite moiety having absorption in this range of wavelengths. Overall, we found a good dependency of both emission spectra versus $\lambda_{\mathrm{ex}}$ and excitation spectra versus $\lambda_{\mathrm{em}}$ and such behaviour has been reported in multiple chromophorefluorophore systems having aromatic and oxidation groups. ${ }^{59}$

Time-resolved fluorescence measurements of G-Fol samples in THF were performed and showed the multiexponential decay kinetics. As reported by Shang et al. ${ }^{60}$ the origin of fluorescence in graphite oxide material is the recombination of electron-hole from the bottom of the conduction band and nearby localized states to wide-range valence band; moreover, GO emission is predominantly suggested to originate from the 


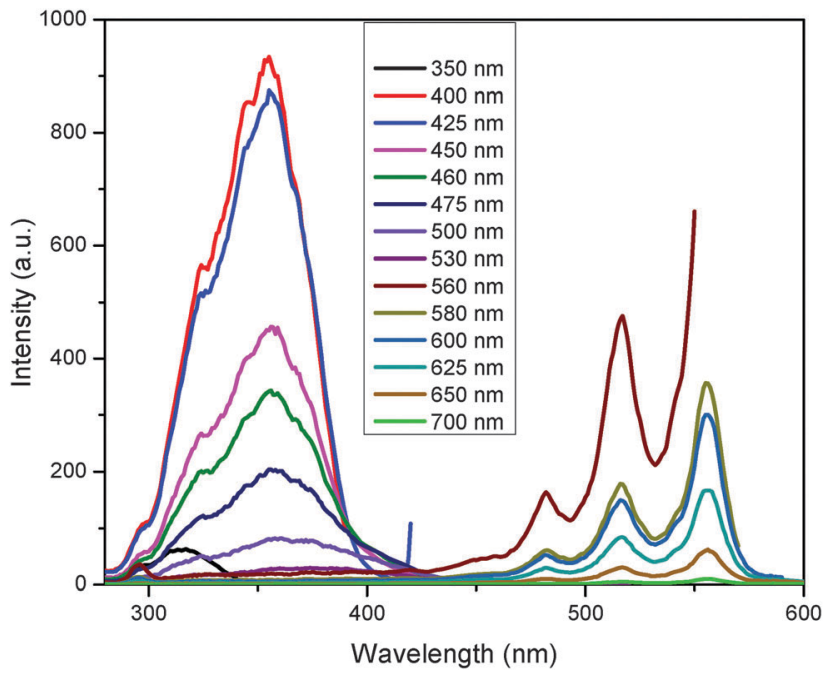

Fig. 10 Excitation fluorescence spectra of G-Fol in THF at different emission wavelengths.

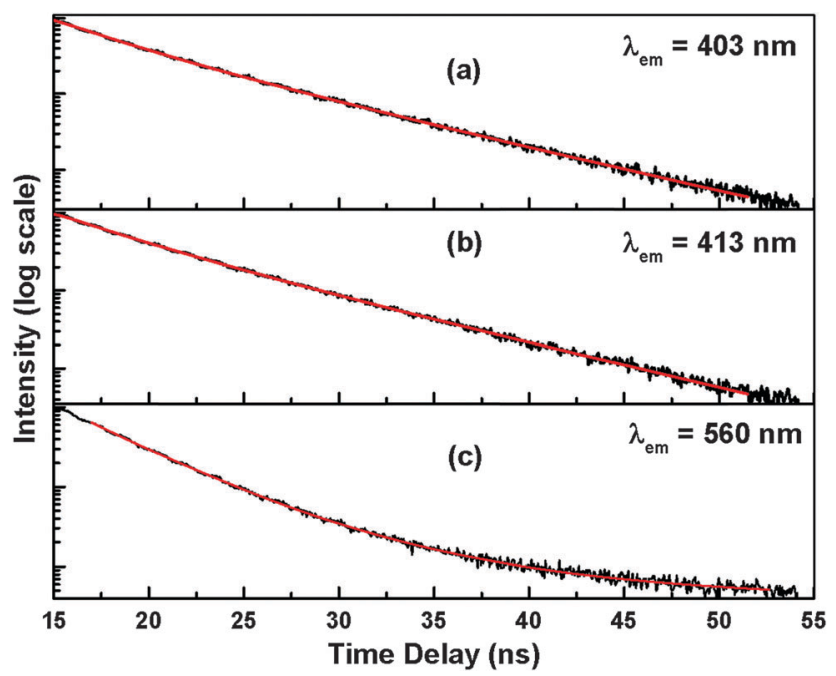

Fig. 11 (a-c) TCSPC measurements of G-Fol in THF at $\lambda_{\text {exc }}=280 \mathrm{~nm}$.

electron transition among/between the non-oxidized carbon region and boundary of oxidized carbon atom region. Fullerene intercalated G-Fol sample is also showing similar behaviour. Fig. 11a-c show the TRF decay curves for G-Fol samples at $\lambda_{\text {ext }}=280 \mathrm{~nm}$ by time-correlated single photon counting (TCSPC) system for $\lambda_{\mathrm{em}}=403,413$ and $560 \mathrm{~nm}$. The kinetic curves show multiexponential decay with three time constants to fit the curves as listed in Table 1. Huge improvement in

Table 1 Time constants with their relative amplitudes to fit the TRF decay curves for G-Fol

\begin{tabular}{lllll}
\hline$\lambda_{\mathrm{ex}} / \mathrm{nm}$ & $\lambda_{\mathrm{em}} / \mathrm{nm}$ & $\begin{array}{l}\tau_{1} \text { (relative } \\
\text { amplitude) } / \mathrm{ns}\end{array}$ & $\begin{array}{l}\tau_{2} \text { (relative } \\
\text { amplitude) } / \mathrm{ns}\end{array}$ & $\begin{array}{l}\tau_{3} \text { (relative } \\
\text { amplitude) } / \mathrm{ns}\end{array}$ \\
\hline 280 & 403 & $3.2(23.72)$ & $0.25(3.8)$ & $7.2(72.48)$ \\
280 & 413 & $3.9(57.35)$ & $0.8(12.6)$ & $9.1(30.05)$ \\
280 & 562 & $0.62(0.38)$ & $3.68(79.9)$ & $9.4(19.72)$
\end{tabular}

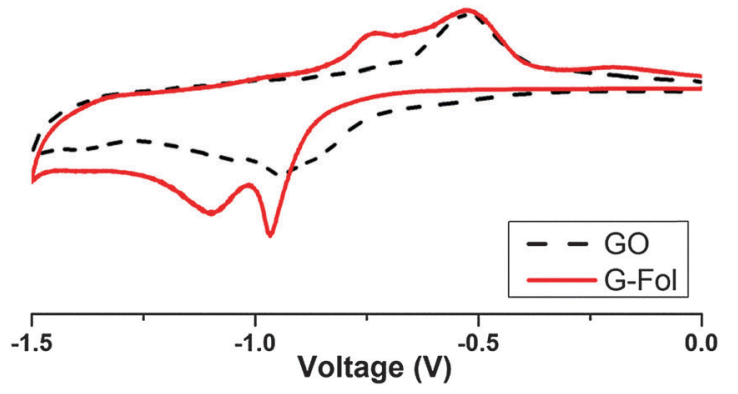

Fig. $12 \mathrm{CV}$ of GO and G-Fol samples recorded as films in acetonitrile using TBAPF $_{6}$ as supporting electrolyte.

emission lifetimes has been observed compared to previously reported data for graphene oxide. ${ }^{60}$

The electrochemical properties of the hybrid compound G-Fol were also investigated by cyclic voltammetry (CV). For comparison, electrochemical behaviour of GO was also recorded. All experiments were performed in films at room temperature using acetonitrile as solvent and tetrabutyl ammonium hexafluorophosphate $\left(\mathrm{TBAPF}_{6}\right)$ as supporting electrolyte. The first reduction potential for $\mathrm{GO}$ was observed at $-0.91 \mathrm{~V}$, which is also reversible. On the other hand, G-Fol shows two reversible reduction potentials at -0.97 and $-1.1 \mathrm{~V}$. The chemical modification by attachment of fullerenol molecules on graphene sheet is causing cathodic shift of first reduction potential of GO (from $-0.91 \mathrm{~V}$ to $-0.97 \mathrm{~V}$ ), suggesting the existence of

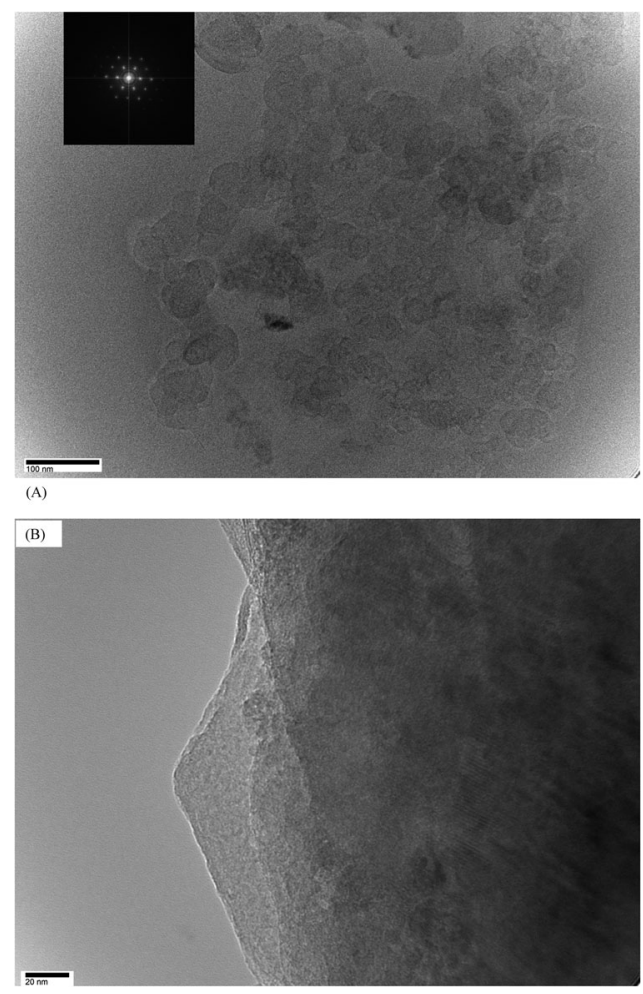

Fig. 13 HRTEM images of G-Fol (A) exfoliated graphite sheets showing graphene flakes (inset: graphene lattice with FFT), and (B) few-layered graphene sheet. 
electronic interaction between the two moieties (Fig. 12).$^{38}$ Moreover, significant shift in reduction onset is observed on functionalization of GO ( $\left.\operatorname{red}_{\text {onset }}=-0.38 \mathrm{~V}\right)$ with fullerenol to form G-Fol $\left(\right.$ red $\left._{\text {onset }}=-0.67 \mathrm{~V}\right)$. This clearly indicates effective change in energy levels of resultant material G-Fol, which is required for device applications. ${ }^{61}$

HRTEM was performed to characterize the exfoliation of graphite interlayer due to intercalation of fullerene molecules (Fig. 13) and formation of stable functionalized graphene sheets. Fig. 13A shows the exfoliated graphene sheets by fullerenol intercalation. The high quality of the sheets with high crystallinity was clearly demonstrated by the perfect crystal lattice in HRTEM image (inset) and the corresponding fast Fourier transform (FFT) with a typical six fold symmetry pattern. Fig. 13B clearly shows the formation of few layer (2-4) graphene flakes; thus, G-Fol makes stable exfoliated graphite, avoiding the restacking of graphene sheets.

\section{Conclusions}

A promising and mild methodology for the preparation of stable exfoliated graphite has been demonstrated by insertion of water soluble fullerenol molecules, providing less chemically defected few layered graphene. We also identified that fullerenol intercalated graphene sheets form long term stable dispersions, i.e., avoiding restacking, in THF and water, which is currently required for preparation of good quality films in devices as buffer layer. Furthermore, work is being carried out to test these materials as buffer layers and also for attachment of different photoactive fullerene derivatives on graphite sheets to explore their application in organic photovoltaics.

\section{Acknowledgements}

Authors acknowledge DST APEX program for funding. The authors are thankful to Prof. R. C. Budhani, Director, CSIR-NPL, for continuous encouragement and support and Dr Rajiv K. Singh for CV measurements. The authors also acknowledge Dr G. D. Sharma and Mr Ramil Bharadwaj for technical support.

\section{References}

1 A. K. Geim and K. S. Novoselov, The rise of graphene, Nat. Mater., 2007, 6, 183-191.

2 K. S. Novoselov, A. K. Geim, S. V. Morozov, D. Jiang, Y. Zhang, S. V. Dubonos, I. V. Grigorieva and A. A. Firsov, Science, 2004, 306, 666-669.

3 C. Chung, Y.-K. Kim, D. Shin, S.-R. Ryoo, B. H. Hong and D.-H. Min, Acc. Chem. Res., 2013, 46, 2211-2224.

4 M. Choucair, P. Thordarson and J. A. Stride, Nat. Nanotechnol., 2008, 4, 30-33.

5 Y.-W. Son, M. L. Cohen and S. G. Louie, Phys. Rev. Lett., 2006, 97, 216803.

6 X. Wang, Y. Ouyang, X. Li, H. Wang, J. Guo and H. Dai, Phys. Rev. Lett., 2008, 100, 206803.
7 X. Li, X. Wang, L. Zhang, S. Lee and H. Dai, Science, 2008, 319, 1229-1232.

8 L. A. Ponomarenko, F. Schedin, M. I. Katsnelson, R. Yang, E. W. Hill, K. S. Novoselov and A. K. Geim, Science, 2008, 320, 356-358.

9 M. Y. Han, B. Ozyilmaz, Y. B. Zhang and P. Kim, Phys. Rev. Lett., 2007, 98, 206805.

10 C. Stampfer, J. Güttinger, F. Molitor, D. Graf and K. Ensslin, Appl. Phys. Lett., 2008, 92, 012102.

11 D. V. Kosynkin, A. L. Higginbotham, A. Sinitskii, J. R. Lomeda, A. Dimiev, B. K. Price and J. M. Tour, Nature, 2009, 458, 872-876.

12 K. S. Novoselov, A. K. Geim, S. V. Morozov, D. Jiang, M. I. V. Grigorieva, S. V. Dubonos and A. A. Firsov, Nature, 2005, 438, 197-200.

13 T. Kuila, A. K. Mishra, P. Khanra, N. H. Kimc and J. H. Lee, Nanoscale, 2013, 5, 52-71.

14 I. V. Lightcap and P. V. Kamat, Acc. Chem. Res., 2013, 46, 2235-2243.

15 D. Dodoo-Arhin, M. Fabiane, A. Bello and N. Manyala, Ind. Eng. Chem. Res., 2013, 52, 14160-14168.

16 X. Wang, L. Z. Zhi and K. Mullen, Nano Lett., 2008, 8, 323-327.

17 J. B. Wu, H. A. Becerril, Z. N. Bao, Z. F. Liu, Y. S. Cheng and P. Peumans, Appl. Phys. Lett., 2008, 92, 263302.

18 S. Biswas and L. T. Drzal, Nano Lett., 2009, 9, 167-172.

19 S. Park and R. S. Ruoff, Nat. Nanotechnol., 2009, 4, 217-224.

20 A. Reina, X. Jia, J. Ho, D. Nezich, H. Son, V. Bulovic, M. S. Dresselhaus and J. Kong, Nano Lett., 2009, 9, 30-35.

21 S. Vaziri, G. Lupina, C. Henkel, A. D. Smith, M. Östling, J. Dabrowski, G. Lippert, W. Mehr and M. C. Lemme, Nano Lett., 2013, 13, 1435-1439.

22 V. Ryzhii, M. Ryzhii, A. Satou and T. J. Otsuji, Appl. Phys., 2008, 103, 094510.

23 M. D. Stoller, S. Park, Y. Zhu, J. An and R. S. Ruoff, Nano Lett., 2008, 8, 3498-3502.

24 S. R. C. Vivekchand, C. S. Rout, K. S. Subrahmanyam, A. Govindaraj and C. N. R. Rao, J. Chem. Sci., 2008, 120, 9-13.

25 B. C. Brodie, Ann. Chim. Phys., 1860, 59, 466-472.

26 W. S. Hummers and R. E. Offeman, J. Am. Chem. Soc., 1958, 80, 1339-1340.

27 K. S. Novoselov, V. I. Falko, L. Colombo, P. R. Gellert, M. G. Schwab and K. Kim, Nature, 2012, 490, 192-200.

28 D. R. Dreyer, S. Park, C. W. Bielawski and R. S. Ruoff, Chem. Soc. Rev., 2010, 39, 228-240.

29 S. Eigler, M. E-Heim, S. Grimm, P. Hofmann, W. Kroener, A. Geworski, C. Dotzer, M. Rockert, J. Xiao, C. Papp, O. Lytken, H.-P. Steinruck, P. Muller and A. Hirsch, Adv. Mater., 2013, 25, 3583-3587.

30 M. Quintana, E. Vazquez and M. Prato, Acc. Chem. Res., 2013, 46, 138-148.

31 Z. Q. Wei, D. E. Barlow and P. E. Sheehan, Nano Lett., 2008, 8, 3141-3145.

32 D. Li, M. B. Muller, S. Gilije, R. B. Kaner and G. G. Wallace, Nat. Nanotechnol., 2008, 3, 101-105. 
33 S. Niyogi, E. Bekyarova, M. E. Itkis, J. L. McWilliams, M. A. Hamon and R. C. Haddon, J. Am. Chem. Soc., 2006, 128, 7720-7721.

34 Y. F. Xu, Z. B. Liu, X. L. Zhang, Y. Wang, J. G. Tian, Y. Huang, Y. F. Ma, X. Y. Zhang and Y. S. Chen, Adv. Mater., 2009, 21, 1275-1279.

35 J. R. Lomeda, C. D. Doyle, D. V. Kosynkin, W. F. Hwang and J. M. Tour, J. Am. Chem. Soc., 2008, 130, 16201-16206.

36 C. Xu, X. D. Wu, J. W. Zhu and X. Wang, Carbon, 2008, 46, 386-389.

37 S. Stankovich, R. D. Piner, S. T. Nguyen and R. S. Ruoff, Carbon, 2006, 44, 3342.

38 M. Barrejón, M. Vizuete, M. J. Gómez-Escalonilla, J. L. G. Fierro, I. Berlanga, F. Zamora, G. Abellán, P. Atienzar, J.-F. Nierengarten, H. García and F. Langa, Chem. Commun., 2014, 50, 9053-9055.

39 D. Yu, K. Park, M. Durstock and L. Dai, J. Phys. Chem. Lett., 2011, 2, 1113-1118.

40 Y. Zhang, L. Ren, S. Wang, A. Marathe, J. Chaudhuri and G. Li, J. Mater. Chem., 2011, 21, 5386-5391.

41 M. Chen, H. Zhou, F. Yu, H. Yang, G. Wang, J. He and L. Sun, Nanoscale, 2013, 5, 8359-8362.

42 K. Miura and M. Ishikawa, Materials, 2010, 3, 4510-4517.

43 Z.-B. Liu, Y.-F. Xu, X.-Y. Zhang, X.-L. Zhang, Y.-S. Chen and J.-G. Tian, J. Phys. Chem. B, 2009, 113, 9681-9686.

44 X. Y. Zhang, Y. Huang, Y. Wang, Y. F. Ma, Z. F. Liu and Y. S. Chen, Carbon, 2006, 44, 3342-3347.

45 W. Kwon, Y.-H. Kim, C.-L. Lee, M. Lee, H. C. Choi, T.-W. Lee and S.-W. Rhee, Nano Lett., 2014, 14, 1306-1311.

46 J. Luo, J. Kim and J. Huang, Acc. Chem. Res., 2013, 46, 2225-2234.
47 J. Li, A. Takeuchi, M. Ozawa, X. Li, K. Saigo and K. Kitazawa, Chem. Commun., 1993, 1784-1785.

48 R. Singh and T. H. Goswami, in Fullerene Research Advances, ed. C. N. Kramer, NOVA Science Publishers, NY, 2007, ch. 3, pp. 55-96, entitled Recent Development of Fullerenol Chemistry, ISBN 1-60021-824-5.

49 R. Singh and T. H. Goswami, Synth. Met., 2011, 161, 670.

50 T. H. Goswami, B. Nandan, S. Alam and G. N. Mathur, Polymer, 2003, 44, 3209-3214.

51 L. Xiao, H. Shimotani, N. Dragoe, A. Sugita, K. Saigo, Y. Iwasa, T. Kobayashi and K. Kitazawa, Chem. Phys. Lett., 2003, 368, 738-744.

52 T. H. Goswami, R. Singh, S. Alam and G. N. Mathur, Thermochim. Acta, 2004, 419, 97-104.

53 R. Singh and T. H. Goswami, Thermochim. Acta, 2011, 513, 60-67.

54 J. I. Paredes, S. V-Rodil, A. Martínez-Alonso and J. M. D. Tascon, Langmuir, 2008, 24, 10560-10564.

55 W. Ai, W. Zhaou, Z. Du, Y. Du, H. Zhang, X. Jia, L. Xie, M. Yi and W. Huang, J. Mater. Chem., 2012, 22, 23439-23446.

56 D. Graf, F. Molitor, K. Ensslin, C. Stampfer, A. Jungen, C. Hierold and L. Wirtz, Nano Lett., 2007, 7, 238-242.

57 J. M. Tour, Nat. Mater., 2014, 13, 545-546.

58 S. You, S. M. Luzan, T. Szabo and A. V. Talyzin, Carbon, 2013, 52, 171-180.

59 F. V. Subach and V. V. Verkhusha, Chem. Rev., 2012, 112, 4308-4327.

60 J. Shang, L. Ma, J. Li, W. Ai, T. Yu and G. G. Gurzadyan, Sci. Rep., 2012, 2(792), 1-8.

61 N. Wang, L. Sun, X. Zhang, X. Bao, W. Zheng and R. Yang, RSC Adv., 2014, 4, 25886-25891. 